\title{
COMPARAÇÃO ENTRE BLOCOS CERÂMICOS PRODUZIDOS EM FORNOS HOFFMAN E CAIEIRA *
}

\section{Resumo}

\author{
Euzébio Bernabé Zanelato ${ }^{1}$ \\ Jonas Alexandre ${ }^{2}$ \\ Afonso Rangel Garcez Azevedo 3 \\ Markssuel Teixeira Marvila ${ }^{4}$ \\ Sergio Neves Monteiro ${ }^{5}$ \\ Niander Aguiar Cerqueira ${ }^{6}$ \\ Gustavo de Castro Xavier ${ }^{7}$
}

A produção de peças cerâmicas pela indústria da cerâmica vermelha é composta por diferentes processos, dentre eles, se destaca o processo de queima. O processo de queima é um dos principais responsáveis pelas propriedades finais da peça cerâmica, tais como: resistência mecânica e porosidade. Por ser um processo gradativo, onde a temperatura é elevada até determinado grau, o ambiente proporcionado pelo forno é fundamental para a homogeneidade de queima das peças, no entanto, existem diferentes formas para a realização dessa queima. $O$ objetivo deste projeto é comparar e avaliar o processo de queima por forno Hoffman e forno Caieira. Para caracterização da argila foram realizados os ensaios de granulometria, densidade real dos grãos, limites de Atterberg e análise química. Para acompanhamento da temperatura do forno foi utilizado um termopar digital e verificada a temperatura durante o processo de queima. Para avaliação do melhor processo de queima foram realizados os ensaios de variação linear e absorção de água das peças cerâmicas. Os resultados indicaram que as peças cerâmicas queimadas pelo forno Hoffman apresentam propriedades superiores aos do forno Caieira.

Palavras-chave: Tijolos cerâmicos; Hoffman; Caieira; Processo de queima.

\section{COMPARISON BETWEEN CERAMIC BLOCKS PRODUCED IN HOFFMAN AND CAIEIRA OVENS.}

\section{Abstract}

The production of ceramics by the red ceramic industry is composed of different processes, among which stands out the burning process. The burning process is one of the main responsible for the final properties of the ceramic part, such as: mechanical strength and porosity. Because it is a gradual process, where the temperature is raised to a certain degree, the environment provided by the furnace is fundamental for the homogeneity of burning of the pieces, however, there are different ways to perform this burning. The objective of this project is to compare and evaluate the firing process by Hoffman oven and Caieira oven. To characterize the clay were conducted test of particle size, real density of the grains, Atterberg limits and chemical analysis. To monitor the oven temperature was used a digital thermocouple and checked the temperature during the firing process. For better evaluation of the process of burning the linear variation tests were performed and the water absorption of the ceramic pieces. The results indicated that the ceramic pieces burned by the Hoffman oven present superior properties to the Caieira oven.

Keywords: Ceramic bricks; Hoffman; Caieira; Burning process.

Engenheiro Civil, doutorando em Estruturas, LECIV, Universidade Estadual do Norte Fluminense Darcy Ribeiro, Campos dos Goytacazes, RJ, Brasil. 
2 Engenheiro Civil, doutor em Estruturas, LECIV, Universidade Estadual do Norte Fluminense Darcy Ribeiro, Campos dos Goytacazes, RJ, Brasil.

3 Engenheiro Civil, doutorando em Estruturas, LECIV, Universidade Estadual do Norte Fluminense Darcy Ribeiro, Campos dos Goytacazes, RJ, Brasil.

4 Engenheiro Civil, mestrando em Estruturas, LECIV, Universidade Estadual do Norte Fluminense Darcy Ribeiro, Campos dos Goytacazes, RJ, Brasil.

5 Engenheiro Metalúrgico, doutor em Ciência e Engenharia de Materiais, Seção de Engenharia Mecânica e de Materiais, Instituto Militar de Engenharia, Rio de Janeiro, RJ, Brasil.

6 Engenheiro Civil, doutorando em Estruturas, LECIV, Universidade Estadual do Norte Fluminense Darcy Ribeiro, Campos dos Goytacazes, RJ, Brasil.

7 Engenheiro Civil, doutor em Estruturas, LECIV, Universidade Estadual do Norte Fluminense Darcy Ribeiro, Campos dos Goytacazes, RJ, Brasil, 


\section{INTRODUÇÃO}

A indústria da cerâmica vermelha é responsável por parte considerável da economia local no município de Campos dos Goytacazes, por este motivo, estudos realizados com o intuito de avaliar o processo para propor melhorias e aumentar a eficiência do processo de fabricação são fundamentais. Como verificado por outros trabalhos, a disparidade tecnológica entre as indústrias cerâmicas locais são evidentes, o que evidencia a necessidade de algumas cerâmicas se atualizarem para que seus produtos não sejam inferiores aos produzidos por outras cerâmicas.

Analizando o processo de fabricação das peças cerâmicas, verifica-se que a etapa de queima destaca-se como uma das principais etapas. O processo de queima, de forma geral, é similar em todas as cerâmicas locais, onde o procedimento é dado pelo aquecimento gradativo dos fornos por longos períodos e elevação final da temperatura até o patamar de queima, no entanto, devido a utilização de diferentes fornos, a eficiência com que cada forno realiza a queima é diferente.

A verificação da eficiência de diferentes tipos de fornos é recorrente, trabalhos como Vieira (2002) que entre os fornos utilizados encontra-se o Hoffman, ou de Morais (2010) que entre os fornos utilizados encontra-se o Caieira, além do relatório produzido pelo INT que apresentou dados comparativos entre diversos fornos. Vale ressaltar também os estudos realizados com o objetivo de avaliar individualmente cada etapa do processo de fabricação para cada tipo de forno, como o de Lima (2002) que analisou o forno Caieira e o de Lima (2002) que analisou o forno Hoffman.

Os fornos Hoffman e Caieira foram escolhidos neste trabalho devido ao seu recorrente uso no município de Campos, conforme comprovado pelo diagnostico de Ramos (2006), onde foi verificado que os dois principais fornos utilizados são o Hoffman e o Caieira.

Este trabalho tem como objetivo comparar o processo de queima de peças cerâmicas em fornos Hoffman e Caieira, assim será possível verificar se um dos fornos é mais eficiente e quanto mais eficiente o mesmo é.]

\section{MATERIAIS E MÉTODOS}

A argila utilizada no estudo foi coletada pela cerâmica e encontra-se em utilização. Foi utilizada a mesma argila em todas as peças cerâmicas fabricada para o estudo, onde a mesma foi caracterizada pelos ensaios de distribuição granulométrica, densidade real dos grãos e análise química.

Serão utilizados dois tipos de fornos para a queima das peças cerâmicas conformes descritos a seguir.

\section{- Forno Hoffman}

O forno Hoffman apresenta as seguintes medidas: 12,90m (largura) $\times 91,40 \mathrm{~m}$ (comprimento) x $3,50 \mathrm{~m}$ (altura). A capacidade volumétrica do forno é de $10,60 \mathrm{~m}^{3} \mathrm{em}$ cada uma das 15 faixas para o preenchimento com peças cerâmicas. A figura 1 ilustra a morfologia dos fornos. 


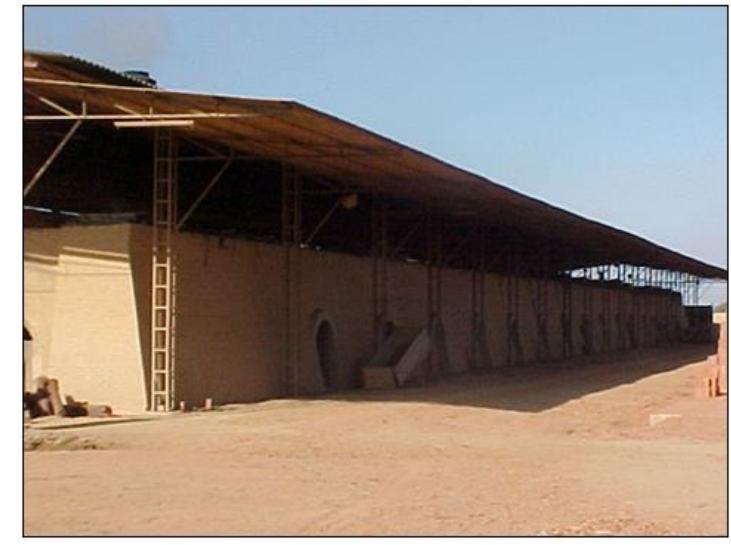

(a)

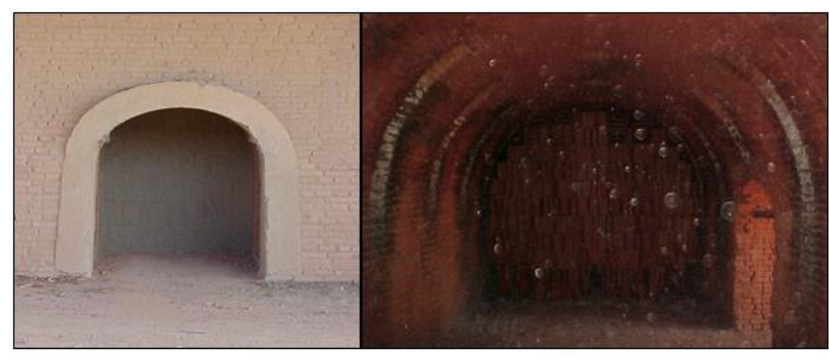

(b)

Figura 1 - Forno Hoffman (a) Vista lateral (b) Vista frontal.

- Forno Caieira

O forno Caieira apresenta as seguintes medidas: 4,45m (largura) $x \quad 5,50 \mathrm{~m}$ (comprimento) x 4,30m (altura). A capacidade volumétrica do forno é de $56,89 \mathrm{~m}^{3}$ para o preenchimento com peças cerâmicas. A figura 2 ilustra a morfologia dos fornos caieira.

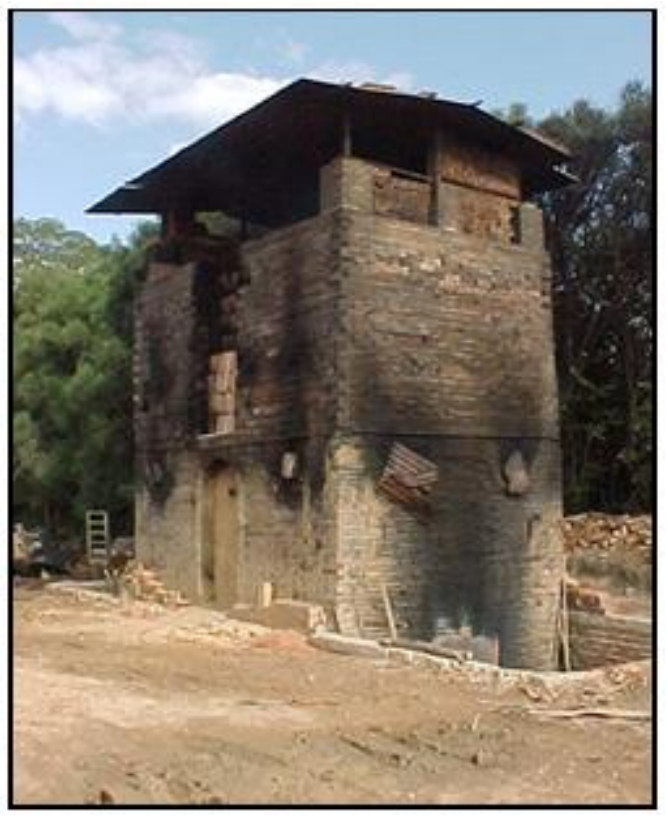

Figura 2 - Forno Caieira.

Para comparar o desempenho da etapa de queima de cada um dos fornos, foram selecionados treze amostras para cada tipo de peça cerâmica, sendo elas, tijolo maciço, tijolo vazado e bloco cerâmico. Serão realizados ensaios de variação linear e absorção de água. Além disso, também será realizado o acompanhamento da temperatura de queima em cada forno com o auxílio de um termopar digital. 


\section{RESULTADOS E DISCUSSÃO}

A distribuição do solo apresentou $60,8 \%$ de argila e $32,2 \%$ de sílte, o que representa um total de $93 \%$ do solo apenas nessas duas faixas granulométricas.

Tabela 1. Distribuição granulométrica da argila.

\begin{tabular}{|c|c|c|c|c|c|c|c|c|}
\hline \multirow{3}{*}{ Amostra } & \multicolumn{8}{|c|}{ Porcentagens Granulométricas } \\
\hline & \multicolumn{3}{|c|}{ Pedregulho } & \multicolumn{3}{|c|}{ Areia } & \multirow{2}{*}{ Sílte } & \multirow{2}{*}{ Argila } \\
\hline & Grosso & Médio & Fino & Grossa & Média & Fina & & \\
\hline Solo & - & - & - & 0,2 & 0,4 & 6,4 & 32,2 & 60,8 \\
\hline
\end{tabular}

Os resultados da análise química da argila estão indicados na Tabela 2.

Tabela 2. Caracterização Química do Resíduo utilizado

\begin{tabular}{|c|c|c|c|c|c|c|c|c|}
\hline Argila & $\mathrm{SiO}_{2}$ & $\mathrm{Fe}_{2} \mathrm{O}_{3}$ & $\mathrm{Al}_{2} \mathrm{O}_{3}$ & $\mathrm{~K}_{2} \mathrm{O}$ & $\mathrm{TiO}_{2}$ & $\mathrm{SO}_{3}$ & $\mathrm{CaO}$ & Outros \\
\cline { 2 - 7 } & 44,97 & 10,74 & 38,22 & 3,1 & 1,46 & 1,1 & 0,31 & 0,1 \\
\hline
\end{tabular}

Os resultados obtidos nos ensaios de densidade real dos grãos e dos limites de Atterberg estão indicados na tabela 3.

Tabela 3. Densidade real dos grãos e limites de Atterberg.

\begin{tabular}{|c|c|}
\hline & Argila \\
\hline LL & 72 \\
\hline LP & 27,3 \\
\hline IP & 44,7 \\
\hline Yg & 2,61 \\
\hline USCS & $\mathrm{CH}$ \\
\hline
\end{tabular}

A Figura 3 ilustra os resultados obtidos pelo acompanhamento da temperatura de queima de cada um dos fornos. Como pode ser observado, ambos fornos apresentam um longo período de pré-aquecimento. O forno caieira apresentou um maior tempo de execução da queima, devido à menor taxa de subida da temperatura até o patamar. 


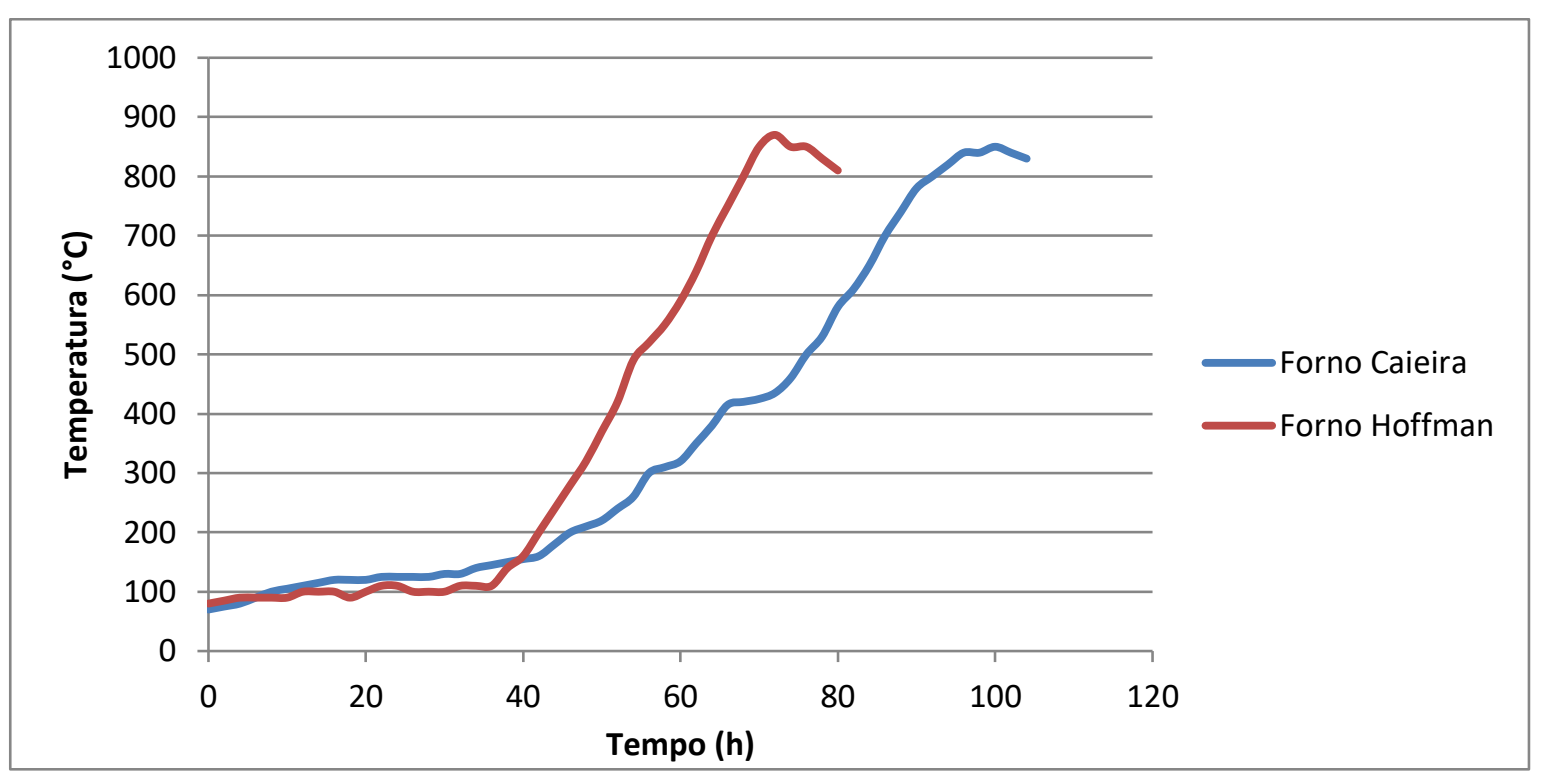

Figura 3. Acompanhamento da temperatura de queima dos fornos.

Os ensaios de variação linear foram realizados em cada uma das dimensões de cada uma das amostras de cada tipo de peça cerâmica. As dimensões selecionadas estão ilustradas na Figura 4.
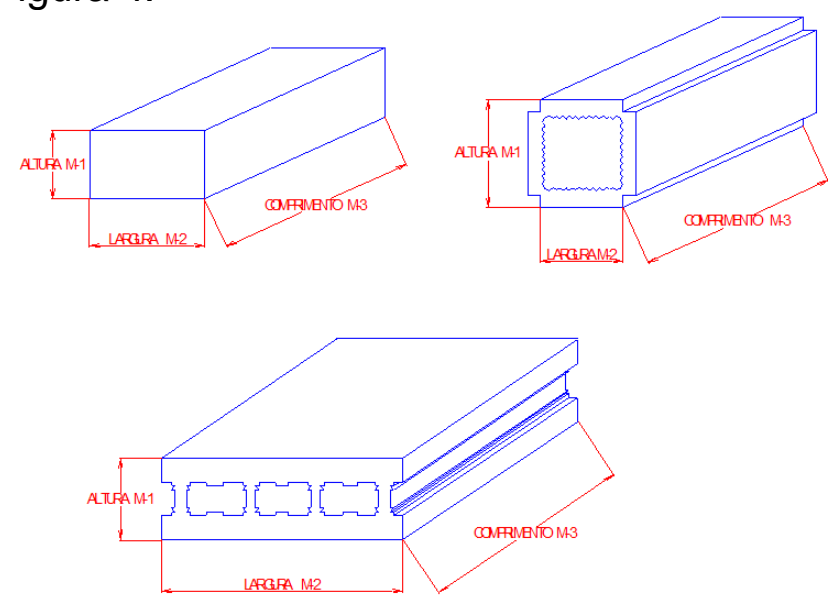

Figura 4. Dimensões das peças cerâmicas.

Os resultados obtidos no ensaio de variação linear da Tabela 4, indicam uma maior variação linear nos tijolos do Forno Hoffman. O que é justificado pela maior velocidade de queima e devido ao ligeiro aumento do valor da temperatura de patamar obtido pelo forno Hoffman.

Tabela 4. Variação Linear.

\begin{tabular}{|c|c|c|c|c|c|c|c|c|c|}
\hline \multicolumn{10}{|c|}{ Variação Linear (\%) } \\
\hline \multirow{2}{*}{ Tipo de forno } & \multicolumn{3}{|c|}{ Tijolo vazado } & \multicolumn{3}{c|}{ Tijolo maciço } & \multicolumn{3}{c|}{ Bloco cerâmico } \\
\cline { 2 - 10 } & M1 & M2 & M3 & M1 & M2 & M3 & M1 & M2 & M3 \\
\hline Forno Caieira & 5,6 & 5,8 & 6 & 5,1 & 5,4 & 5,5 & 6,8 & 6,9 & 6,9 \\
\hline Forno Hoffman & 6,1 & 6,4 & 6,5 & 6,1 & 6,2 & 6,3 & 7,2 & 7,2 & 7,4 \\
\hline
\end{tabular}

Os resultados obtidos pelo ensaio de absorção de água, apontam as peças queimadas pelo forno Hoffman como menos absorventes, conforme pode ser observado na Tabela 5. 
Tabela 5. Absorção de água.

\begin{tabular}{|c|c|c|c|}
\hline \multicolumn{4}{|c|}{ Absorção de água (\%) } \\
\hline Tipo de forno & Tijolo vazado & Tijolo maciço & Bloco cerâmico \\
\hline Forno Caieira & 19,4 & 12,4 & 22,9 \\
\hline Forno Hoffman & 17,8 & 10,2 & 21,1 \\
\hline
\end{tabular}

\section{CONCLUSÃo}

Pode-se concluir que o Forno Hoffman apresentou resultados superiores aos obtidos pelo forno Caieira, onde foi observada maior variação linear e, consequentemente, menor absorção de água pelas peças cerâmicas. O seu desempenho pode ser justificado pela circulação de ar quente internamente do forno Hoffman proporcionar melhores condições para as peças.

Apesar de não ter obtido resultados tão inferiores, é justificável a substituição dos fornos Caieira por fornos Hoffman para aumentar a qualidade das peças cerâmicas fabricadas.

\section{REFERÊNCIAS}

1 I. S. Ramos, M. G. Alves, J. Alexandre. Diagnostico do Polo Cerâmico de Campos dos Goytacazes. Cerâmica Industrial. 2006; 28-32.

2 A. L. Lima, I. Ramos, J. Alexandre. Avaliação do processo de queima em forno Modelo Caieira. Congresso Brasileira de Cerâmica. São Paulo. 2002.

3 A. L. Lima, I. Ramos, J. Alexandre. Avaliação do processo de queima em forno Modelo Hoffman. Congresso Brasileira de Cerâmica. São Paulo - SP. 2002.

4 D. S. G. Morais, J. U. L. Mendes, R. M. Silva, M. O. T. Filho, M. A. V. Cavalcante. Análise comparativa da perda de calor entre o forno Caieira e forno abóbada com isolamento. VI Congresso Nacional de Engenharia Mecânica. Campina Grande - PB. 2010.

5 C. M. F. Vieira, T. M. Soares, S. N. Monteiro. Massa cerâmicas para telhas : características e comportamento de queima. Cerâmica. V. 49. 2003. p. 245-250.

6 Instituto Nacional de Tecnologia - INT. Avaliação do setor de cerâmica da região Norte do Estado do Pará - regiões de Abaetetuba, Igarapé-Miri e São Miguel do Guamá. Rio de Janeiro - RJ. 2011. 